\title{
On the sloshing effect in half-filled freight trains during braking
}

\author{
Jian Dai ${ }^{1}$, Mengmeng $\operatorname{Han}^{2, *}$, Kok Keng Ang ${ }^{1}$, and Minh Thi Tran $^{3}$ \\ ${ }^{1}$ Department of Civil \& Environmental Engineering, National University of Singapore, Singapore \\ ${ }^{2}$ School of Civil Engineering, University of Queensland, St Lucia, Queensland 4072, Australia \\ ${ }^{3}$ Faculty of Civil Engineering, Ho Chi Minh City University of Technology, Vietnam
}

\begin{abstract}
This paper is concerned with a computationally efficient numerical study on the dynamic responses of a half-filled freight train during braking phase using the moving element method. The motion of liquid inside the freight tank is modelled based on potential flow theory. The vehicle components are represented by interconnected rigid multibodies and the track is modelled by an Euler beam resting on a Pasternaktype foundation. The accuracy of the proposed computational model is examined by comparison with available results in the literature. This paper also investigates the effect of the initial train speed and the braking torque on the dynamic response of the train-track system.
\end{abstract}

\section{Introduction}

Railway is a major means of transporting large quantities of freight over long distances. With the modern advancement in railway technology, train speed has been raised several times over the past decades which leads to a great advantage in terms of transportation efficiency. However, there also arises safety concerns over freight trains carrying flammable liquid products. Records of recent accidents have already sent alarming messages to rail designers and operators. This includes the Viareggio train derailment in 2009 and Lac-Mégantic rail disaster in 2013. Thus, there is an imperative need to review the dynamic behaviour of freight trains carrying liquid products.

When liquid products are being transported using freight tanks, the filling level is governed by the allowable axle load and pressure of the vapor. In many cases, these tanks are partially filled, leaving a free surface of the liquid. This gives rise to liquid sloshing phenomenon under dynamic excitations. The sloshing phenomenon may be well captured by using computational fluid dynamics (CFD) approach [1], which however requires a significant amount of computational effort. Thus, many researchers have adopted a reduced mechanical model either in the form of a pendulum system [2, 3] or a mass-spring system [1], which can reduce the computational cost significantly. Unfortunately, this reduced mechanical model requires calibration of the parameters according to CFD simulation or experimental results every time when there is a change in the tank geometry and/or liquid filling level.

\footnotetext{
*Corresponding author: mengmeng.han@uq.net.au
} 
If violent non-linear sloshing phenomenon is not expected, the linear potential flow theory is usually applicable. The potential flow theory assumes ideal incompressible, inviscous and irrotational flow where efficient analytical and numerical solutions are available. Such solutions have been found to be able to predict the sloshing phenomenon with sufficient degree of accuracy [4].

In this paper, we present a computational study on the dynamic response of a half-filled liquid freight train during braking phase. The liquid inside the freight tank is idealized as a linear velocity potential model. The vehicle components are represented by rigid multibodies interconnected by means of spring-dashpot units representing the suspension systems. The track is modelled as an Euler beam supported by a two-parameter Pasternaktype foundation. The accuracy of the proposed sloshing tank model is first examined by comparison with available results in the literature. Then, a 16-degree-of-freedom liquid freight train model and the track model is put forward to investigates the effect of the initial train speed and the braking torque on the dynamic response of the train-track system.

\section{Methodology and formulations}

\subsection{Train-track model}

Consider a freight train that starts braking at a speed $V_{0}$ and with a constant braking torque $T_{b}$, as shown in Figure 1. The train comprises one car body of mass $m_{c}$, pitch moment of inertia $J_{c}$ and length $L$ resting on two bogies of identical mass $m_{b}$ and pitch moment of inertia $J_{b}$ through the secondary suspension system consisting of two spring-dashpot units of spring constant $k_{s}$ and damping coefficient $c_{s}$ each. The bogies are supported through primary suspensions to the four wheels, each of mass $m_{w}$ and moment of inertia $J_{w}$. The primary suspension system consists of four spring-dashpot units of spring constant $k_{p}$ and damping coefficient $c_{p}$. The rail is modelled as a Euler beam of elastic modulus $E$, second moment of inertia $I$ and mass per unit length $m$. The rail is resting on a two-parameter viscously damped Pasternak-foundation of stiffness constant $k$, shear modulus $k_{s m}$, damping coefficients of foundation $\alpha$ and its shear layer $\lambda$, respectively. The train and the track are coupled together through wheel-rail interaction. Nonlinear Hertz contact theory [5] is adopted to model the normal wheel-rail contact. The tangential adhesion forces $f_{i}(i=1,2$, $3,4)$ is calculated based on Polach friction model [6]. In addition, the train is subject to running resistance due to aerodynamic drag and rolling resistance [7]. In view of the symmetry, it is reasonable to consider only one rail supporting half of the train weight. Due to the page constraint, the formulations of the train-track model are omitted. However, one may refer to the authors' earlier published work [8] for more details.

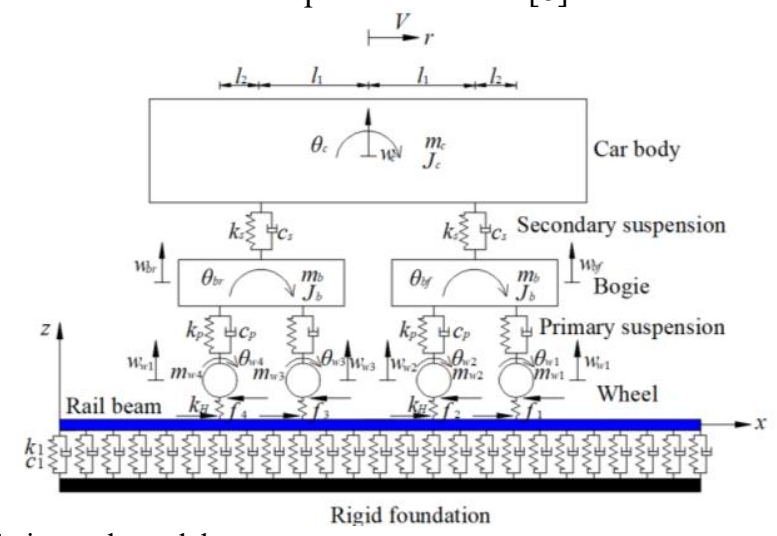

Figure 1. Train-track model. 


\subsection{Liquid sloshing model}

Consider a freight tank that is partially filled with liquid product. The liquid is assumed to be incompressible, inviscous and irrotational so that the linear velocity potential $\phi(x, z, t)$ is applicable. Accordingly, the velocity potential must satisfy the Laplace equation in the fluid domain, solid wall boundary conditions at the fluid-tank interface, linearized kinematic and dynamic free surface boundary conditions at the mean water surface level as well as the mass conservation rule. By assuming the free surface elevation $\eta(x, t)$ in the form of $\beta(t) \cos (\pi(x+L / 2) / L)$ and in view of the abovementioned boundary conditions, the following relationship can be found [4]

$$
\ddot{\beta}+\omega^{2} \beta+\frac{\lambda_{11}}{\mu_{1}}\left(\ddot{u}_{c}-g \theta_{c}\right)+\frac{\lambda_{021}}{\mu_{1}} \ddot{\theta}_{c}=0
$$

where $\beta$ is the temporal function corresponding to the assumed sloshing mode; $\omega$ the fundamental sloshing frequency; $\ddot{u}_{c}$ and $\theta_{c}$ the translational acceleration of the car body (i.e. the storage tank) in the $x$-axis and the pitching rotation, respectively; $\lambda_{11}, \lambda_{021}$ and $\mu_{1}$ the hydrodynamic coefficients to be determined by the added mass properties. The frequency dependent added mass properties corresponding to a sloshing tank with arbitrary geometry can be conveniently computed using commercial boundary element solvers such as WAMIT and HydroSTAR. The liquid force and moment about the tank bottom centre can then be evaluated using the following equations

$$
\begin{gathered}
F_{l}(x)=m_{l}\left(-\ddot{u}_{c}-\ddot{\theta}_{c} z_{c}\right)-\lambda_{11} \ddot{\beta} \\
F_{l}(z)=-m_{l} g-m_{l} \ddot{w}_{c} \\
M_{l}(y)=m_{l}\left(g z_{c} \theta_{c}-z_{c} \ddot{u}_{c}\right)-\left(\lambda_{021} \ddot{\beta}-g \lambda_{11} \beta\right)
\end{gathered}
$$

where $m_{l}$ is the mass of the liquid product inside the storage tank; $g$ the gravitational acceleration; and $z_{c}$ the distance from the mass centre of the liquid to the bottom centre of the tank. The analysis of a partially filled freight train can be conveniently carried out by inclusion of the abovementioned four equations in the global equations of motion of the coupled train-track system.

\subsection{Moving element method}

The moving element method (MEM) is employed for the dynamic analysis of the traintrack system. In principle, this numerical method enjoys the advantages of having the moving loads always located at the same nodal points of the track structure and the moving loads will never travel to the boundary of the selected domain and beyond. Consequently, relatively coarser mesh configuration and smaller domain size can be employed to achieve computational efficiency without compromising the accuracy of the results. In addition, there is also no need for constant tracking and updating of the locations of the moving loads. Compared to the conventional finite element method (FEM), this numerical approach has demonstrated its superiority in terms of both accuracy and efficiency when dealing with various train-track dynamic problems [8-12]. Again, the mathematical formulations are omitted due to the page constraint. Interested readers may refer to the abovementioned references for more details. 


\section{Numerical verification}

Consider a horizontally placed cylindrical tank of radius $R=1.2 \mathrm{~m}$ and length $L=5.75 \mathrm{~m}$. The tank is partially filled with water of density $\rho=998.2 \mathrm{~kg} / \mathrm{m}^{3}$ at two filling levels, namely $h=R$ and $1.5 R$. The tank is experiencing a constant deceleration of $a=0.2 \mathrm{~g}$ along the longitudinal $x$-axis. Note that all these parameters are the same as those reported by Kolaei et al. [13].

Table 1 lists the maximum normalized liquid forces $F_{x}$, which is a ratio of the total liquid forces $F_{l}$ to the forces corresponding to the rigid body motion $F_{x R}=-m_{l} \ddot{u}_{c}$ (see Eq. (2)). Note that the computed hydrodynamic coefficients are $\omega=1.65 \mathrm{rad} / \mathrm{s}, \lambda_{11}=-16466 \mathrm{~kg}$, $\lambda_{021}=-15147 \mathrm{kgm}$ and $\mu_{1}=25434 \mathrm{~kg}$ for $h=R$, and $\omega=2.1 \mathrm{rad} / \mathrm{s}, \lambda_{11}=-14231 \mathrm{~kg}, \lambda_{021}=-$ $20046 \mathrm{kgm}$ and $\mu_{1}=13571 \mathrm{~kg}$ for $h=1.5 R$. The maximum normalized liquid forces reported in [13] are also listed in Table 1 for the purpose of comparison. As can be seen, both results are in excellent agreement with each other, thereby validating the accuracy of the current liquid sloshing model.

Table 1. Maximum resultant liquid forces.

\begin{tabular}{|c|c|c|}
\hline Filling level & Method & Maximum $\boldsymbol{F}_{\boldsymbol{x}}$ \\
\hline \multirow{2}{*}{$h=R$} & Present & 1.47 \\
\cline { 2 - 3 } & Kolaei et al. [13] & 1.47 \\
\hline \multirow{2}{*}{$h=1.5 R$} & Present & 1.29 \\
\cline { 2 - 3 } & Kolaei et al. [13] & 1.29 \\
\hline
\end{tabular}

\section{Numerical study}

The dynamic response of a freight train subject to an abrupt braking is next investigated. The parameters reported in [8] are adopted for the track model. In addition, the train with a tare wagon weight $m_{c}=30.5$ ton, internal diameter $R=1.5 \mathrm{~m}$ and length $L=15.5 \mathrm{~m}$ is employed to represent a Tagkks tank [1]. The tank is half filled with gasoline of density $\rho=$ $830 \mathrm{~kg} / \mathrm{m}^{3}$. Table 2 lists the hydrodynamic coefficients for a full liquid sloshing model.

Table 2. Hydrodynamic coefficients.

\begin{tabular}{|c|c|c|c|}
\hline Parameter & Value & Parameter & Value \\
\hline$\omega$ & $0.69 \mathrm{rad} / \mathrm{s}$ & $\lambda_{11}$ & $137071 \mathrm{~kg}$ \\
\hline$\lambda_{021}$ & $-68461 \mathrm{kgm}$ & $\mu_{1}$ & $442163 \mathrm{~kg}$ \\
\hline
\end{tabular}

Figure 2(a) shows the wheel-rail normal contact forces for a freight train with an initial speed $V_{0}=80 \mathrm{~km} / \mathrm{h}$ subject to an abrupt constant braking torque $T_{b}=5 \mathrm{kNm}$ after $0.5 \mathrm{~s}$ steady-state response. As a comparison, Figure 2(b) shows the contact forces for a freight with an equivalent solid mass but without sloshing effects. As can be seen, the dynamic normal contact forces are subject to large oscillations when there is liquid inside the tank. It is also found that the lead and trailing wheels under the bogie in front are subject to larger forces than their counterparts under the bogie at the rear due to the inertia effect of the liquid during train braking. The normal contact force developed on the leading wheel is also found to be larger than that on the trailing wheel under the same bogie. This is due to the moment arising from the developed tangential contact forces. 


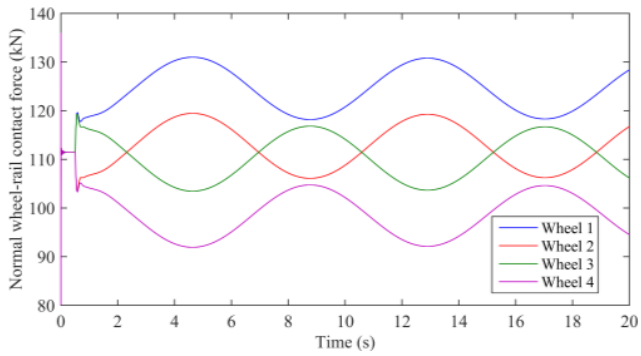

(a)

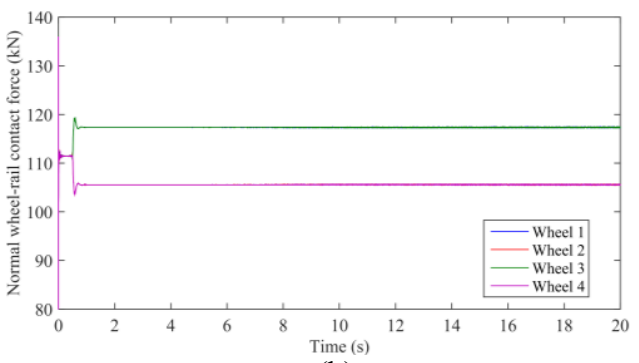

(b)

Figure 2. Wheel-rail contact forces: (a) tank with liquid and (b) tank with equivalent solid mass.

Figures 3(a) and 3(b) show the braking distances under different braking torques for initial train speed $V_{0}=80 \mathrm{~km} / \mathrm{h}$ and $160 \mathrm{~km} / \mathrm{h}$, respectively. For comparison, the cases involving a train with equivalent solid mass are also considered. As can be seen in Figure 3, the most effective braking torque for both initial train speeds is in the range of $15 \mathrm{kNm}$ to $20 \mathrm{kNm}$. It is also found that the liquid sloshing phenomenon tends to increase the braking distance, especially when the initial speed is high and/or the braking torque is large. A $15 \%$ increase is observed when $V_{0}=160 \mathrm{~km} / \mathrm{h}$ and $T_{\mathrm{b}}=20 \mathrm{kNm}$. In view of this, it may be concluded that the existence of free surface inside the storage tank would appreciably hinder the emergency braking of a freight train and thus it should be accounted for in the design and operation of a freight train.

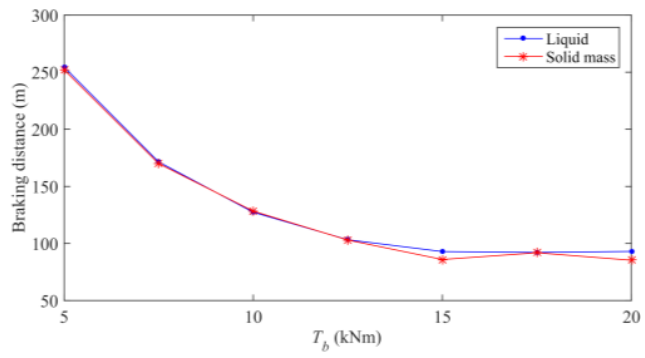

(a)

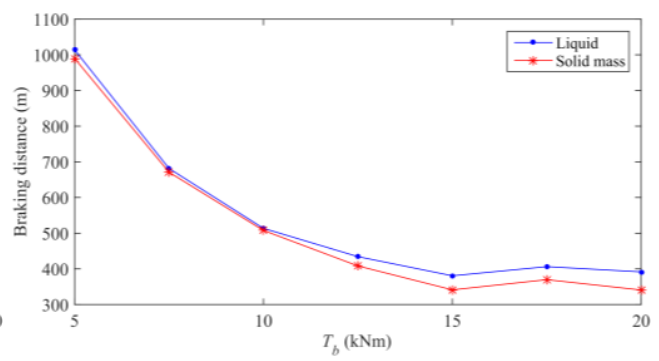

(b)

Figure 3. Train braking distance: (a) $V_{0}=80 \mathrm{~km} / \mathrm{h}$ and (b) $V_{0}=160 \mathrm{~km} / \mathrm{h}$.

\section{Conclusions}

In this paper, a computational model for the response analysis of partially filled freight train subject to abrupt braking is developed. The train is represented by a moving sprung-mass model and the liquid inside the tank is modelled based on the potential flow theory. The track is modelled as an Euler rail beam resting on a Pasternak-type foundation. The train and the track are coupled together by means of wheel-rail normal and tangential contact models. The moving element method is employed to account for the longitudinal motion of the train-track system. The accuracy of the present model is validated by comparison with available results in the literature. Results of the parametric study show that the liquid sloshing motion affects the dynamic wheel-rail contact forces significantly. The sloshing also tends to hinder the braking of a train, resulting in a longer braking distance. 


\section{References}

[1] E.D. Gialleonardo, A. Premoli, S. Gallazzi, S. Bruni, Veh. Syst. Dyn., 54, 1715-1735 (2017)

[2] S. Aliabadi, A. Johnson, J. Abedi, Comput. Fluids, 32, 535-545 (2003)

[3] C. Vera, J. Paulin, B. Suarez, M. Gutierrez, Proc. IMechE. Part F: J. Rail Rapid Transit, 219, 245-259 (2005)

[4] O.M. Faltinsen, A.N. Timokha, Sloshing (Cambridge University Press, 2009)

[5] C. Esveld, Modern railway track (MRT Productions, Duisburg, 2001)

[6] O. Polach, Wear, 258, 992-1000 (2005)

[7] C.D. Yang, Y.P. Sun, Int. J. Control, 74, 905-920 (2001)

[8] M.T. Tran, K.K. Ang, V.H. Luong, J. Dai, Veh. Syst. Dyn., 54, 1715-1735 (2017)

[9] K.K. Ang, J. Dai, J. Sound Vib., 333, 2054-2970 (2013)

[10] K.K. Ang, J. Dai, M.T. Tran, V.H. Luong, Int. J. Comput. Methods, 11, 1343007 (2014)

[11] J. Dai, K.K. Ang, M.T. Tran, V.H. Luong, D. Jiang, Proc. IMechE. Part F: J. Rail Rapid Transit, 232, 783-797 (2018)

[12] J. Dai, K.K. Ang, D. Jiang, V.H. Luong, M.T. Tran, Int. J. Struct. Stab. Dyn., 18 (2018) https://doi.org/10.1142/S0219455418501225

[13] A. Kolaei, S. Rakheja, M.J. Richard, Eur. J. Mech. B/Fluids, 53, 251-263 (2015) 